\title{
Modelos de brotes arbustivos 0 algas en arquitectura. O cómo replicar un vegetal mediante la Agregación Limitada por
} Difusión (DLA)

\author{
Salvador Serrano Salazar ${ }^{1}$; José Carrasco Hortal ${ }^{2}$; Francesc Morales Menárguez ${ }^{3}$ \\ 1. salvaserrano31@gmail.com \\ 2. Universidad de Alicante, Departamento de Expresión Gráfica y Cartografía, Escuela Politécnica Superior, Ali- \\ cante, España. jose.carrasco@ua.es \\ 3. morales.francesc@gmail.com
}

\begin{abstract}
Resumen
En el presente artículo se expone el desarrollo de un método de diseño de estructuras ramificadas del tipo algas marinas o formas arbustivas que se basa en la agregación limitada por difusión (DLA) para definir su geometría. Se ha usado la DLA para reproducir unas reglas de crecimiento convincentes o verosímiles a partir de lo aprendido de visores programables como el NetLogo (Wilensky 1999). En concreto, las herramientas que reproducen la simulación aprendida de NetLogo son el software Grasshopper para generar las geometrías, el plug-in Exoskeleton para obtener superficies envolventes a dichas estructuras alámbricas, y el plug-in Weaverbird para suavizar transiciones entre caras de malla. Ésta última herramienta permite suavizar la malla mediante iteraciones que aumentan o no el número de caras, lo que permite entender algunas teorías sobre transiciones suaves en bifurcaciones de estructuras naturales (Mattheck 1990).
\end{abstract}

Este artículo sirve además para reflexionar acerca de cómo modelos físico cinéticos basados en mecanismos inspirados en la Inteligencia Artificial ayudan a compartir métodos de análisis con otras disciplinas como la cibernética o la dinámica de fluidos o las ciencias sociales y del medioambiente. ¿Por qué puede ocurrir esto? Por el rigor en el lenguaje que todo el rato pretende referirse a poblaciones de individuos, a ciclos de vida, a sistemas multivariables, a reglas de reciprocidad o a pactos con partículas próximas.

Palabras clave: agregación limitada por difusión, modelos naturales. estructuras, crecimientos, diseño asistido por algoritmos

\begin{abstract}
This article discusses the development of a design method for branched structures with seaweed-like or shrub-like forms based on diffusion-limited aggregation (DLA) to define its geometry. DLA has been used to reproduce convincing or credible growth rules from what has been learned from programmable displays such as NetLogo (Wilenski 1999). In particular, the tools that reproduce the simulation learned from NetLogo are the Grasshopper software to generate the geometry, the Exoskeleton plug-in to get surrounding surfaces to these wireframe structures, and the Weaverbird plug-in to smooth transitions between mesh faces. This last tool allows smoothing the mesh by iterations that increase or not the number of faces, which allows to understand some theories about smooth transitions in forks of natural structures (Mattheck 1990).

This article also serves to reflect on how kinetic-physical models based on mechanics inspired by Artificial Intelligence help to share methods of analysis with other disciplines such as cybernetics or fluid dynamics or the social and environmental sciences. Why can this happen? Because of the rigor in language that all the time tries to refer to populations of individuals, to life cycles, to multi-variable systems, to reciprocity rules or to pacts with near particles.
\end{abstract}

Key words: diffusion-limited aggregation, natural models. structures, growth, algorithm-aided design 


\section{Introducción}

Hace ya tiempo que han surgido prácticas dentro de la arquitectura que se pueden interpretar desde principios de los sistemas complejos o de la llamada Ciencia de la Complejidad: lo deseable es que, en primer lugar, la arquitectura aprenda de cómo el universo sabe auto-organizarse y, en segundo lugar, entienda que es imposible explicar un proceso morfológico de un modo lineal y predecible (Jencks 1997). La arquitectura ha sido capaz de incorporar estos sistemas inestables interpretando modelos evolutivos naturales que sugieren formas de reproducirlos parametrizando su regularidad, variabilidad y adaptabilidad (Holland 1992 y Frazer 2009). Trabajar en parámetros de un sistema complejo emergente es lo que se llama también modelado basado en agentes (Fromm 2004). En otras disciplinas relacionadas con la arquitectura, como la ecología o la sociología, también se ha introducido el uso de estos métodos.

El punto de partida de este trabajo lo constituyen las formas ingrávidas de algunos tipos de algas o las formas de brotes arbustivos (Fig. 1). ¿Por qué nos fijamos en estos modelos? Pues porque los modelos agregativos permiten cuestionar cada fase de diseño; además, trabajar con parámetros ayuda a aislar escalas de aproximación o de fractalidad diversas, como cuando un sistema estructural complejo puede discretizarse en pórticos u otros planos de análisis para entender la transferencia de esfuerzos entre acciones y entorno.
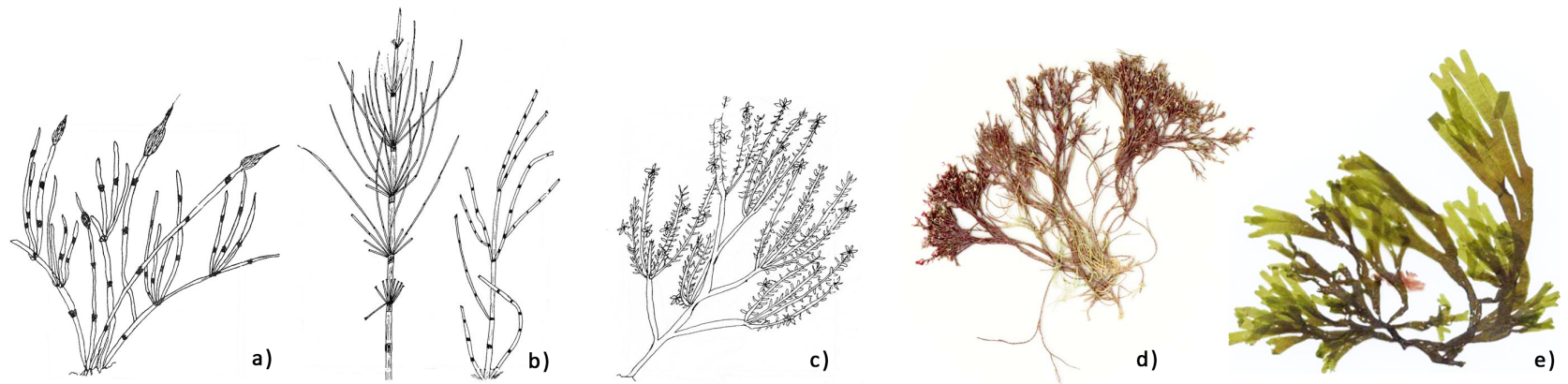

Fig. 1. Canutillo (Equisetum hyemale $L$ ) (a y b); hierba del pasmo (c); alga Jania Rubens (d); alga dictyota (e)

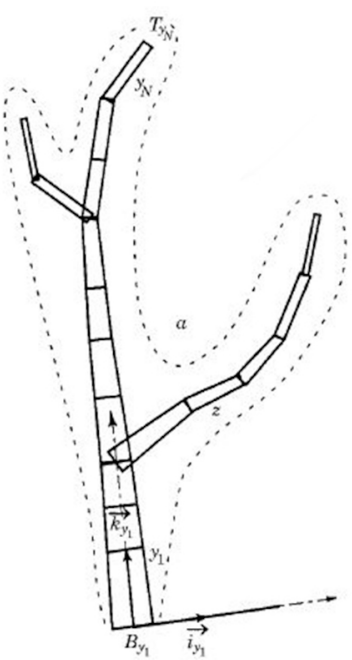

a)
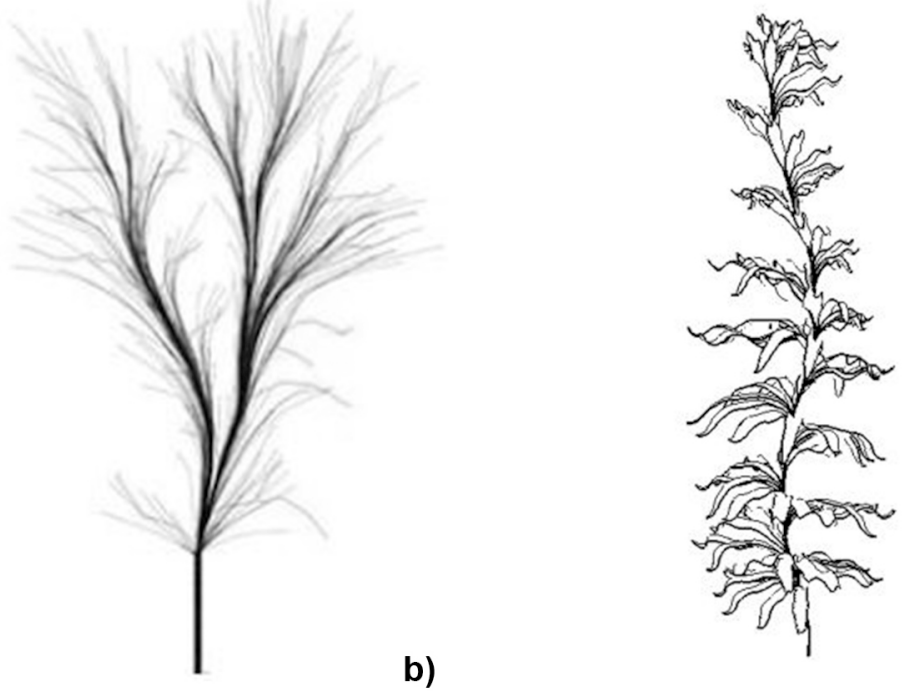

c)

Fig. 2. Descripción de la arquitectura de una planta integrando topología y geometría (Godin et al. 1999) (a); modelado de árbol usando flujo de partículas (Neubert et al. 2007) (b); método de representación de plantas a partir de bocetos (Anastacio et al. 2006) (c).

Las plantas son un ejemplo de estructuras complejas presentes en la naturaleza. Existen varios métodos para comprimir sus diferentes niveles de información y aproximarse a su representación, desde una perspectiva global o desde una perspectiva modular (Godin 2000). Unos de los sistemas de modelizado de estructuras arbóreas más populares y estudiados son los L-Systems, los cuales permiten la generación de naturalezas recursivas auto-similares a partir de un conjunto de reglas gramaticales aplicadas iterativamente (Prusinkiewickz et al.1990). Otros modelos representan las plantas basándose en la información topológica y geométrica de su arquitectura 
(Godin et al. 1999) o incorporan a sus dinámicas de crecimiento los efectos de su propio peso y geometría (Yan et al. 2004).

Como alternativa a estos métodos que se centran en la morfogénesis de las plantas basándose en reglas, se han propuesto otros procedimientos que construyen un modelo tridimensional de una estructura arbórea a partir de imágenes. Estos procesos requieren una muestra en forma de mapa de bits y pueden necesitar de interacción, bien sea para editar la estructura ramificada de la planta o para refinar la segmentación que produce el algoritmo de partición de grafos (Quan et al. 2006), o para determinar el follaje de un árbol del que sólo se tiene su tronco y sus ramas al que se le aplica una simulación de flujo de partículas (Neubert et al. 2007).

Un tercer tipo de métodos de modelado de plantas se basa en la interpretación de bocetos producidos por un usuario con mayor o menor nivel de precisión. Estos métodos presentan un nivel de interacción superior a los anteriores. Pueden partir de un dibujo alámbrico bidimensional que se somete a una serie de procesos que le añaden niveles complejidad (Okabe et al. 2005), o bien requerir la definición morfológica tanto de la estructura del cuerpo de la planta como de sus hojas o pétalos (Anastacio et al. 2006) (Fig. 2).

La geometría de plantas, árboles y naturalezas arbustivas en general ha inspirado el diseño de estructuras a lo largo de la historia de la arquitectura: en primer lugar, debido al interés por el estudio de sus propiedades físicas, mecánicas y biológicas (Gordon 1988, Rian y Sassone 2014); en segundo lugar, debido a que su fractalidad es identificable en todas las fases de crecimiento de tallos a ramas y subramas; finalmente, debido a que los esquemas son fácilmente traducibles a procesos industrializables de barras que bifurcan o derivan después de nudos.

Este interés se ha incrementado en las últimas décadas gracias al desarrollo de métodos de análisis y optimización. Los nuevos métodos derivan de trabajos analógicos pioneros de Frei Otto sobre forma eficaz (form finding) y caminos de fuerzas (optimal paths) (Nerdinger 2005, Otto y Rasch 1995), hasta llegar a la perspectiva analítica actual a través de la optimización con algoritmos genéticos (Buelow 2007) (Fig. 3). En este marco ¿qué parámetros se tendía a establecer en primer lugar?: aquellos que condicionaban la morfogénesis, como el ángulo entre ramas, el número y longitud de ramas y el desplazamiento de nodos en el espacio.
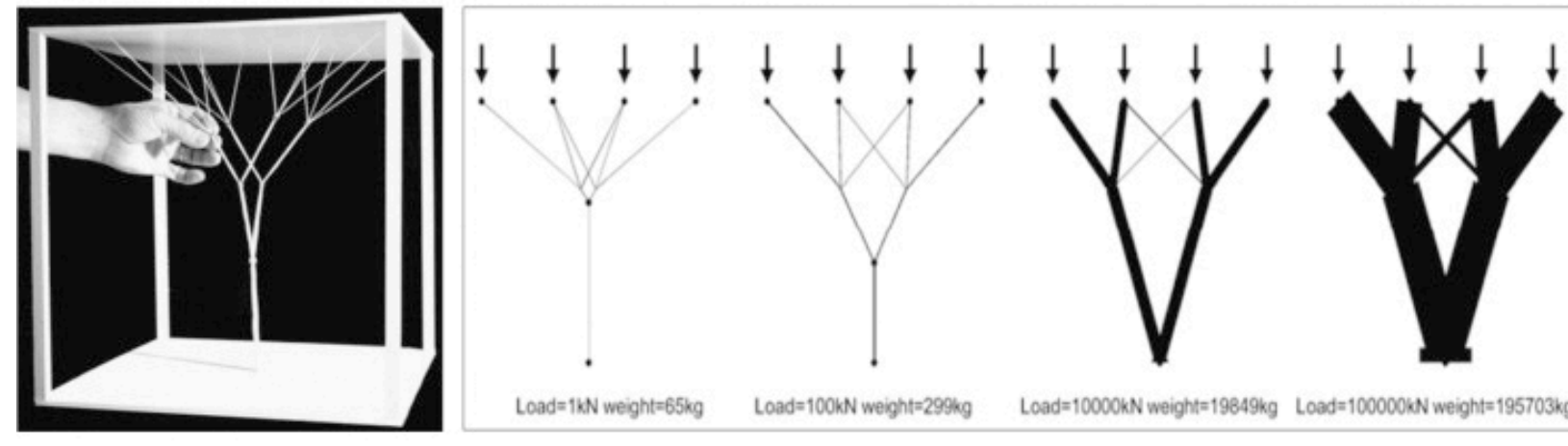

Load=1kN weight=65kg Load=100kN weight $=299 \mathrm{~kg}$

Load=10000 kN weight=19849kg Load=100000kN weight=195703kg
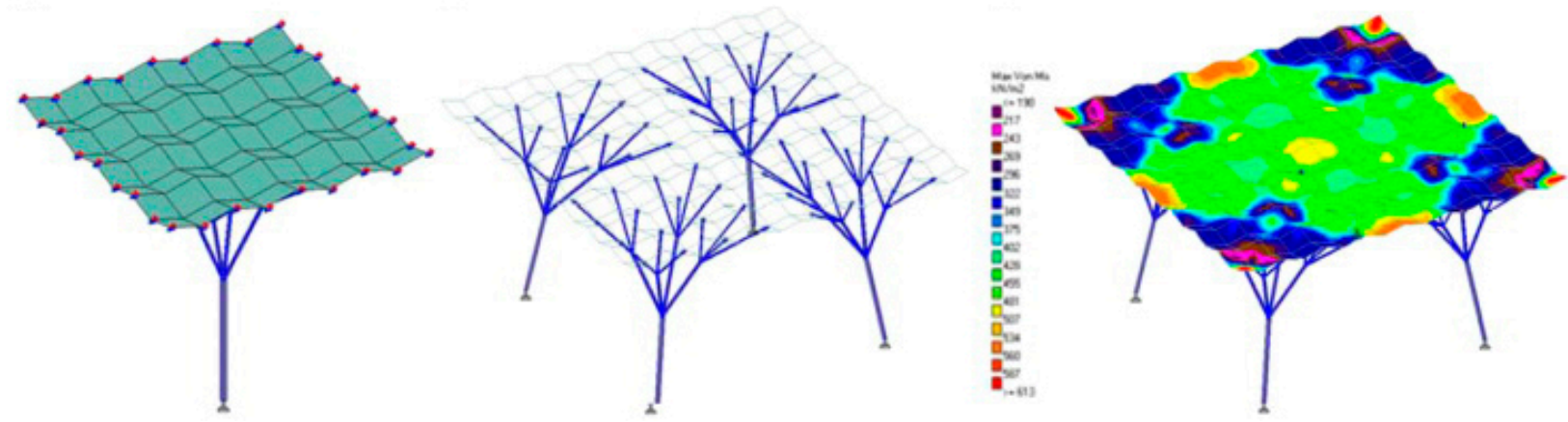

Fig. 3. Modelo con nudos ajustables y niveles de carga (sup) (Buelow, 2007); árboles con placas (inf) (Falk y Buelow, 2009).

Existen algunos ejemplos de aplicaciones de los modelos citados anteriormente como el Qatar National Convention Centre de Arata Isozaki (2011); o el "The Tote Restaurant" de Serie Architects en Bombai (Rian y Sassone 2014) el cual incorpora una estructura de acero diseñada mediante L-Systems (Gawell 2013) (Fig. 4). 


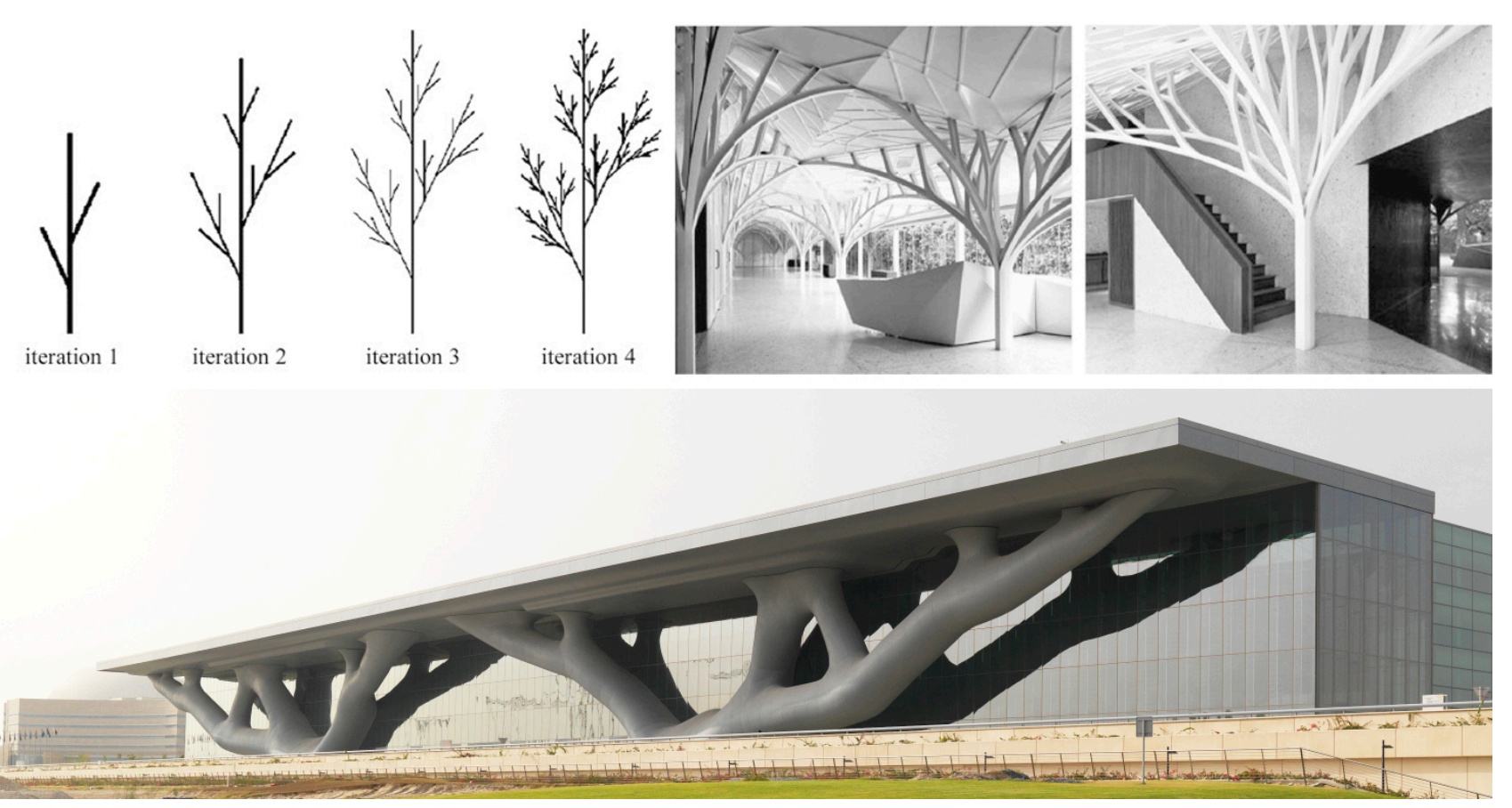

Fig. 4. The Tote Restaurant" de Serie Architects (sup); Qatar National Convention Centre de Arata Isozaki (inf).

Los objetivos de este trabajo son: experimentar con la aplicación de sistemas complejos al diseño arquitectónico; proponer un nuevo modelo de aproximación a la representación de estructuras ramificadas de plantas y algas a partir de la modificación del modelo DLA; ampliar la literatura sobre métodos que relacionan el diseño arquitectónico con geometrías, proporciones o reglas extraídas de especies orgánicas; comparar los resultados del método propuesto con otros procesos de modelizado o proyectos desarrollados recientemente.

\section{Exploración de la agregación limitada por difusión (DLA)}

Se ha partido de la exploración del modelo de agregación limitada por difusión (DLA, por sus siglas en inglés: Diffusion-limited aggregation). EI DLA es un proceso en el que una serie de partículas que se mueven en el espacio de manera aleatoria se van agregando a un cuerpo que las atrapa e inmoviliza, formándose un clúster ramificado (Witten y Sander 1983). Las principales aplicaciones de este patrón de crecimiento se refieren a la descripción de fenómenos físicos. No obstante, el DLA también ha sido relacionado con modelos orgánicos. Existe una relación estadística entre la ramificación lateral del DLA y las hojas de los árboles (Turcotte 1998).

En primer lugar, se han efectuado una serie de simulaciones mediante la aplicación informática NetLogo (Wilensky 1999), que dispone de varios modelos que reproducen este patrón de agregación basándose en la definición de Witter y Sander.

En uno de los primeros ensayos, el DLA se dispersa sobre un espacio bidimensional (Wilensky 1997). Al iniciar la rutina, una serie de partículas (color rojo) comienzan a moverse erráticamente por el espacio bidimensional, mientras que en el centro de éste se sitúa una partícula captora fija en su posición (color verde). Cuando alguna de las partículas en movimiento contacta con la partícula captora su posición queda fijada y pasa a formar parte del cuerpo agregado. A medida que la simulación avanza en el tiempo, más partículas en movimiento se van uniendo al cuerpo agregado y, así, se va conformando el patrón DLA.

La aplicación NetLogo tiene en cuenta las relaciones de vecindad entre las partículas captoras del conjunto agregado y las partículas en movimiento para determinar si estas últimas son atrapadas. El espacio bidimensional está discretizado en una rejilla de píxeles en la que cada partícula ocupa un único píxel. Las partículas en movimiento viajan de píxel en píxel de manera errática. Si en algún momento una partícula en movimiento llega a ocupar algún píxel colindante a una partícula del cuerpo agregado, la primera queda atrapada y pasa a formar 
parte del segundo. Se consideran píxeles colindantes a cualquiera de los que rodean a una partícula del cuerpo agregado, incluyendo las diagonales.

En la segunda simulación, se ha explorado el patrón DLA sobre un espacio bidimensional con partículas sometidas a un movimiento de dirección constante, orientada hacia la primera partícula captora del agregado (Wilensky 2005). A diferencia del primer modelo, en este caso las partículas se desplazan siguiendo una trayectoria recta desde posiciones aleatorias de la periferia hacia el centro del espacio bidimensional en el que opera la aplicación, donde se encuentra la primera partícula captora. Al colisionar con ésta, las partículas en movimiento quedan atrapadas y pasan a formar parte del cuerpo agregado. A medida que el tiempo de la simulación avanza, se van acumulando partículas en el cuerpo agregado y se va formando el patrón DLA.

Otra diferencia con el anterior modelo es que la condición para que una partícula sea agregada no responde a las relaciones de vecindad entre píxeles, sino a la distancia entre el centro de las partículas. Todas las partículas constan del mismo radio $R$. Una partícula en movimiento se agrega a una partícula fija cuando los centros de ambas se encuentran a la distancia de colisión $d$, de modo que se cumpla la expresión $d=2 R$.

En la siguiente simulación, se ha ejecutado un modelo que varía del anterior en que la agregación se desarrolla sobre una línea en vez de sobre una partícula central (Wilensky 2005). Todas las partículas en movimiento siguen una misma dirección perpendicular a la línea captora. Las primeras partículas que colisionan con la línea se agregan a ésta y, a medida que van precipitándose, las partículas en movimiento se van agregando a las previamente depositadas. A medida que avanza el tiempo de simulación, el patrón DLA va emergiendo con una marcada direccionalidad perpendicular a la línea de agregación. La manera en que se produce la colisión de las partículas en movimiento responde a las mismas reglas que el modelo anterior (Fig. 5). 

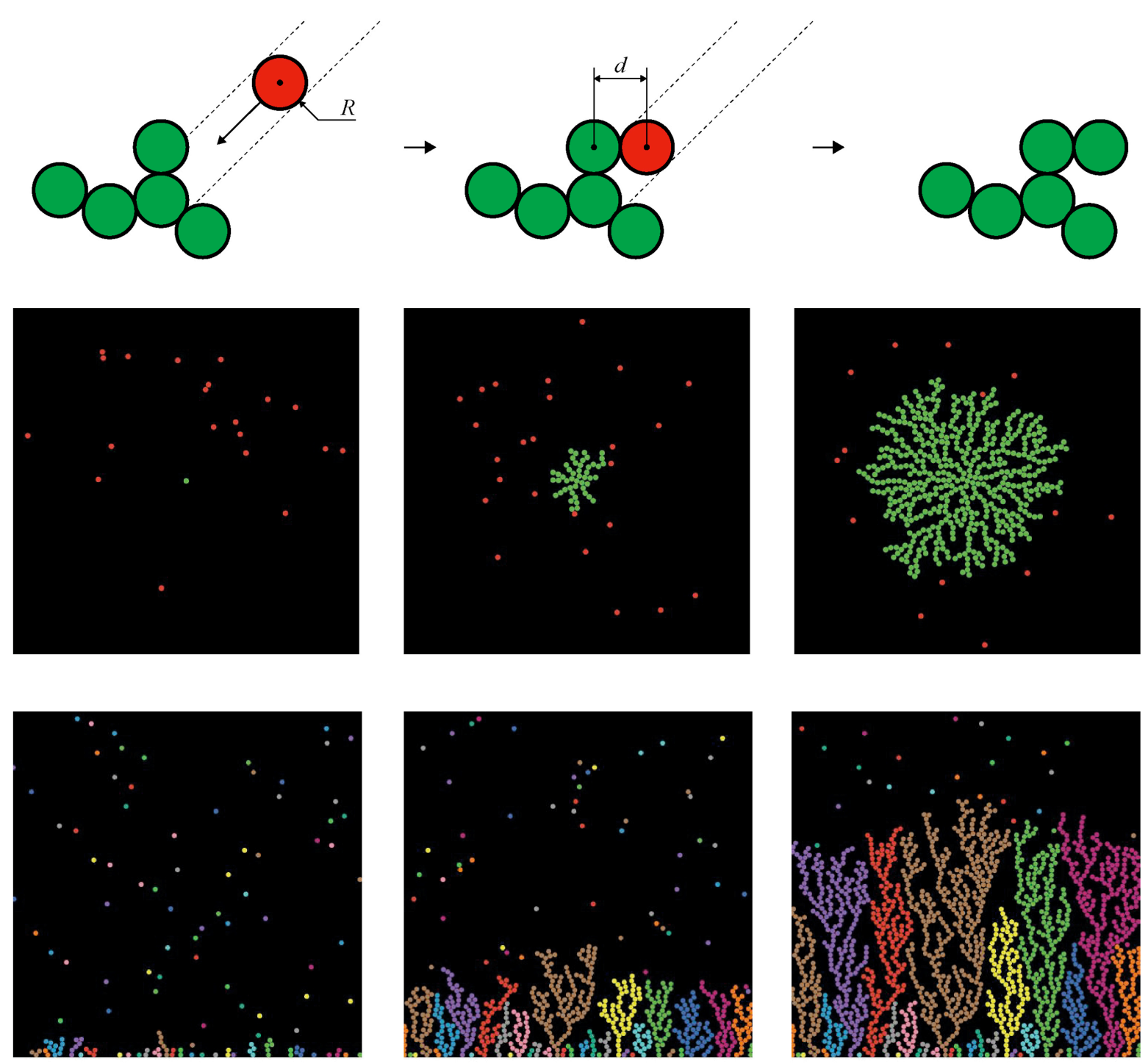

Fig. 5. Colisión y agregación de una partícula en movimiento (sup); fotogramas de la simulación en NetLogo del modelo DLA Alternate (med); fotogramas de la simulación en NetLogo del modelo DLA Alternate Linear (inf)

\section{Modificación del modelo DLA Alternate Linear de NetLogo}

Después de la exploración de los anteriores modelos con el uso de NetLogo, se plantea una modificación del último modelo con el objetivo de profundizar en el potencial de la agregación limitada por difusión como patrón generador de estructuras tridimensionales. Para ello, se ha utilizado el software Rhinoceros y el plug-in de modelado paramétrico Grasshopper como herramientas de programación del modelo modificado.

El primer paso que se ha dado en la modificación del modelo es considerar la estructura alámbrica del patrón DLA en vez del conjunto de círculos que representan las partículas agregadas. De esta manera, se reduce el cuerpo de las partículas a su propio centro, y el patrón DLA pasa a formarse a partir de las líneas que unen estos centros (Fig. 6). 

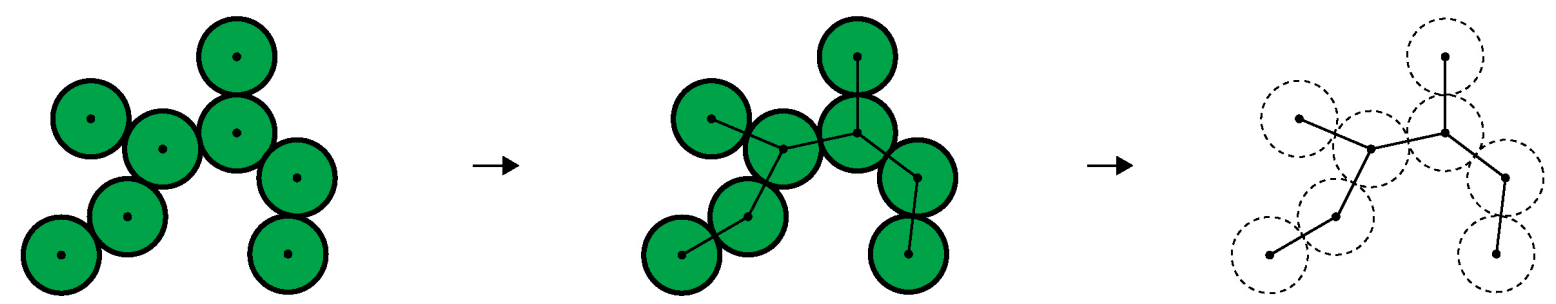

Fig. 6. Reducción del patrón DLA a su estructura alámbrica.

El segundo paso dado en la modificación del modelo es trasladar la acción de la agregación a un espacio tridimensional. En este espacio se sitúa una superficie base sobre la que se distribuyen $n$ partículas captoras en posiciones aleatorias. Las partículas en movimiento se desplazan desde la parte superior del espacio hacia abajo, siguiendo una trayectoria rectilínea perpendicular a la superficie base. La rutina que se ha programado establece que sobre la superficie base se precipitan tandas de $n$ ' partículas en movimiento. En cada tanda, las partículas se desplazan contenidas en un mismo plano paralelo a la superficie base.

Las partículas captoras tienen una distancia de captura $r$, la cual se mide sobre el plano $Q_{i}$ paralelo a la superficie base que contiene a cada una de las partículas. A su vez, el parámetro $d_{i j}$ mide la mínima distancia entre cada partícula captora $i$ y la línea que representa la trayectoria de cada partícula en movimiento $j$. Si $d_{i j}=<r$, entonces la partícula en movimiento $j$ es atrapada por la partícula captora $i$ y pasa a formar parte del cuerpo agregado. Nótese que, siempre que se cumpla la anterior condición, una partícula en movimiento puede ser atrapada por más de una partícula captora, así como una partícula captora puede atrapar más de una partícula en movimiento en la misma tanda. Las partículas en movimiento que no son atrapadas por ninguna partícula captora son descartadas. Al ser capturada, la partícula en movimiento $j$ se detiene sobre un plano $Q_{i}^{\prime}$ paralelo a $Q_{i}$, separado a una distancia $h$ de éste, y se une mediante un segmento de línea con su partícula captora $i$ (Fig. 7). Este segmento de línea pasa a formar parte del patrón DLA. El cociente $h / r$ determina el valor máximo que puede tomar la pendiente de cualquiera de los segmentos del patrón DLA. 

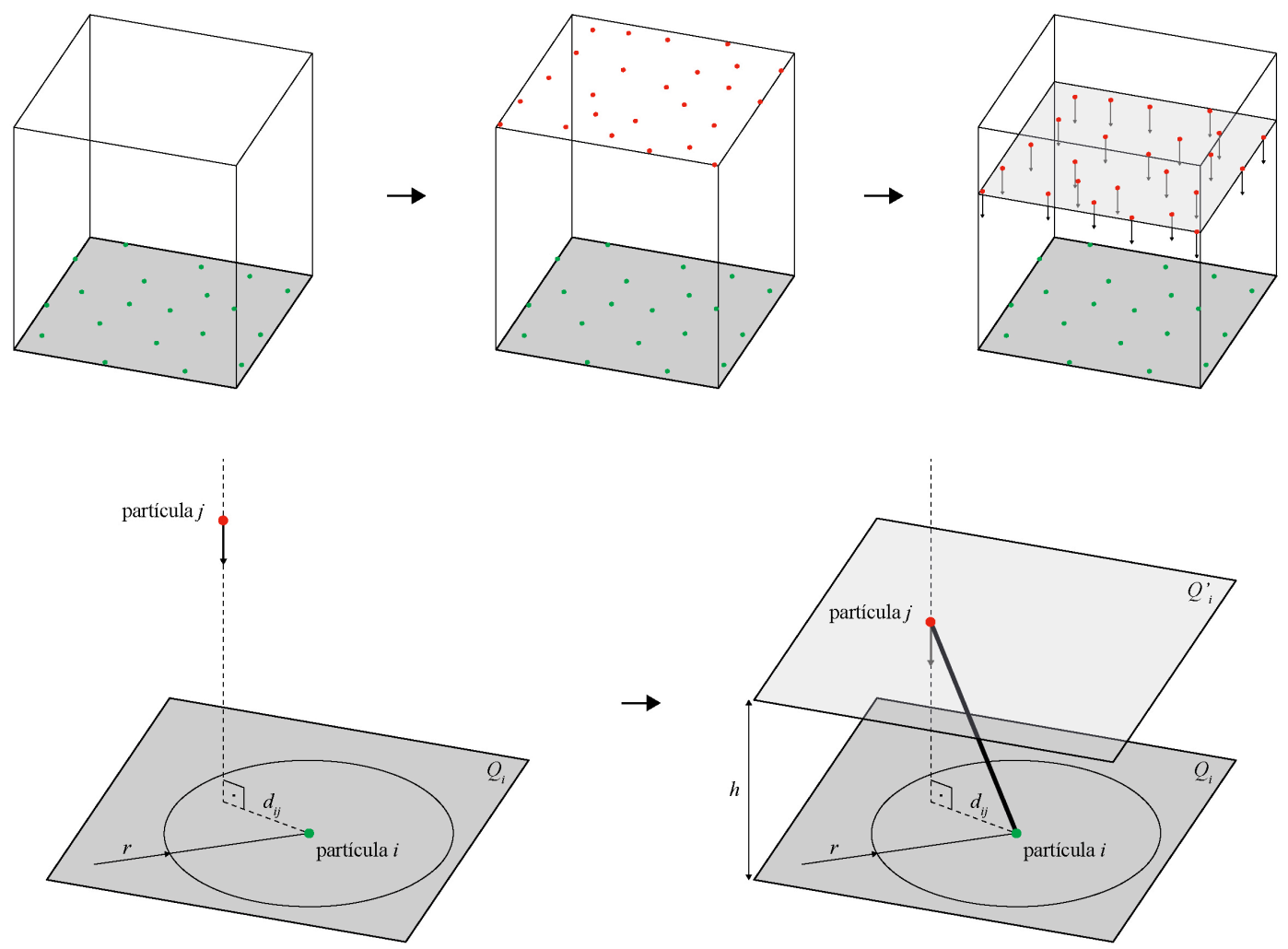

Fig. 7. Primera tanda de partículas en movimiento precipitándose sobre la superficie, para $n=20$ y $n^{\prime}=25$ (sup); y captura de una partícula en movimiento (inf)

La rutina programada se desarrolla durante una cantidad de iteraciones $t$. En cada iteración, se precipita una tanda de partículas en movimiento sobre el cuerpo agregado y éstas van siendo capturadas, convirtiéndose en partículas captoras en la siguiente iteración. A medida que se suceden las iteraciones, el patrón DLA se va conformando, creciendo en dirección perpendicular a la superficie base (Fig. 8). 

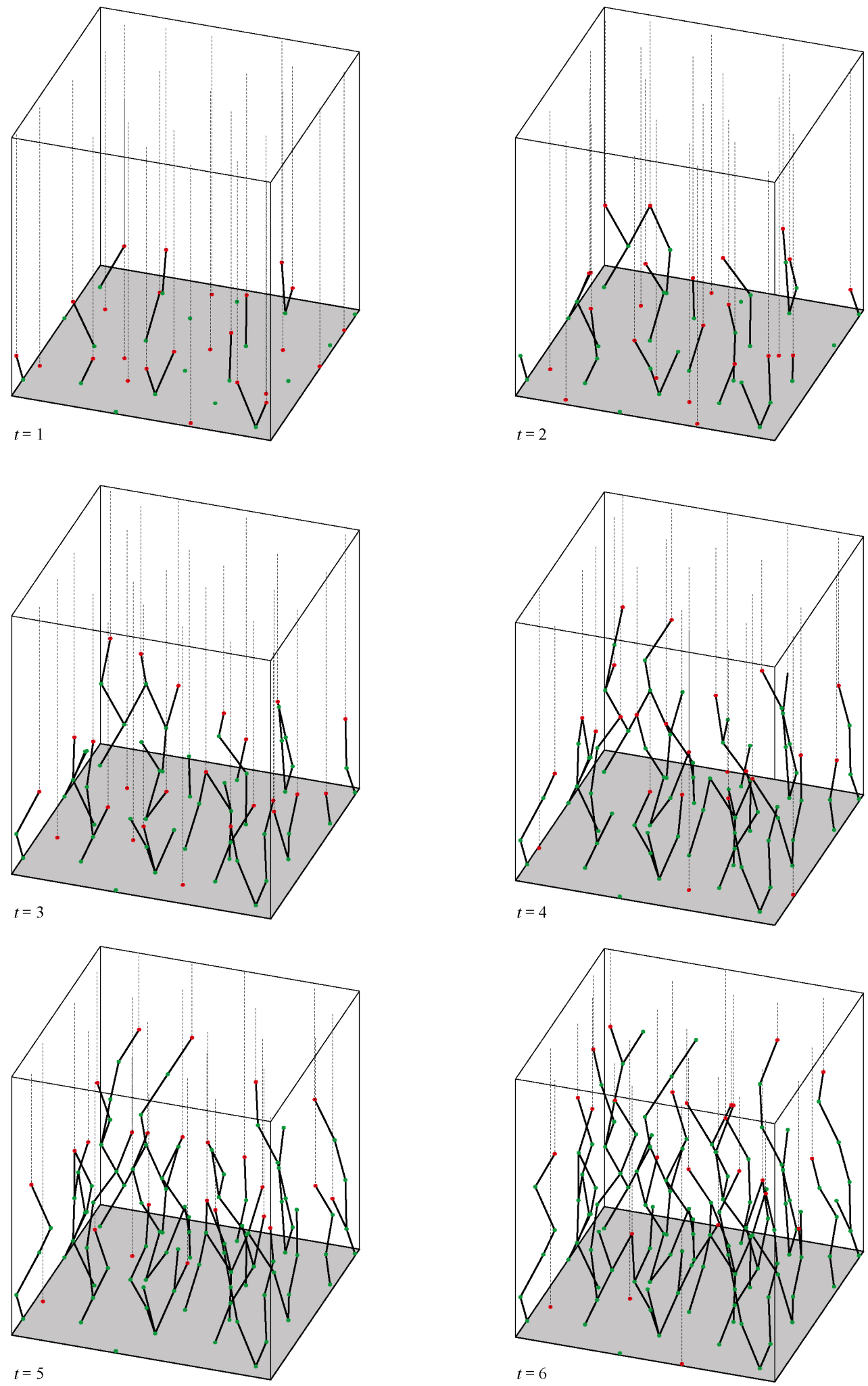

Fig. 8. Formación del patrón DLA modificado durante 6 iteraciones, para $n=20$ y $n^{\prime}=25$

En este punto, se dota de volumen al cuerpo agregado recubriendo la estructura alámbrica obtenida con una malla poligonal en forma de envolvente tubular. Para ello, se emplea el plug-in Exoskeleton, basado en el método Solidifyng Wireframe (Srinivasan et al. 2005). 
El proceso comienza envolviendo los segmentos de línea con tubos cuya directriz es un polígono regular de s lados, circunscrito en una circunferencia de radio $R_{T}$. Estos tubos no llegan a los extremos de los segmentos, sino que se detienen antes para evitar que se produzcan intersecciones indeseadas entre aquellos que confluyen en un mismo nudo. Los polígonos en los que se detienen los tubos se llaman caras finales del tubo. Después, cada nudo se forma computando la envolvente convexa tridimensional de los vértices de las caras finales que se agrupan en torno a dicho nudo. Por último, la unión de los tubos y los nudos compone la malla poligonal que envuelve a la estructura alámbrica (Fig. 9).

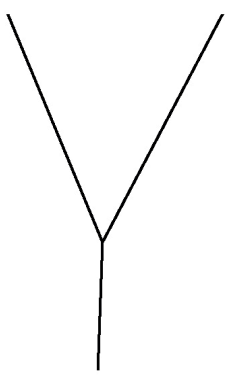

(a)

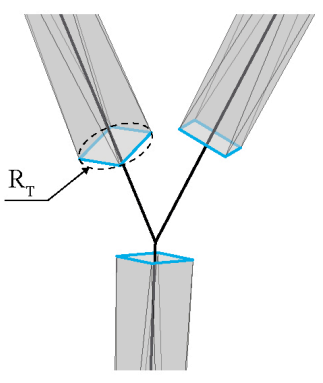

(b)

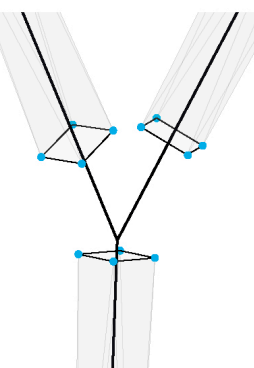

(c)

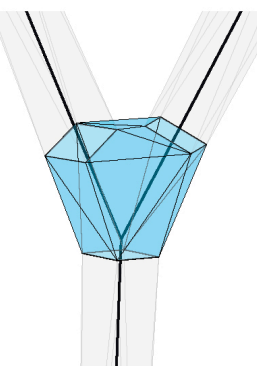

(d)

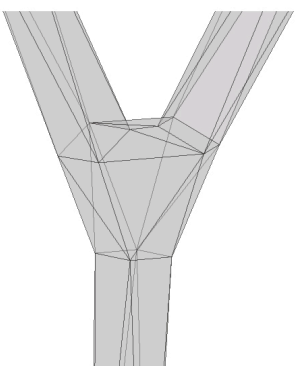

(e)

Fig. 9. Generación de malla poligonal que envuelve estructura alámbrica por el método Solidifyng Wireframe: (a) estructura alámbrica inicial, (b) tubos envolventes para $s=4$ y caras finales, (c) vértices de caras finales, (d) nudo a partir de envolvente convexa tridimensional de vértices de caras finales, y (e) unión de nudo y tubos formando malla poligonal envolvente.

Después de construir la envolvente de la estructura alámbrica, empleamos el plug-in Weaverbird, que contiene herramientas para la edición de mallas poligonales, para aplicar dos operaciones de suavizado. La primera de ellas es el suavizado laplaciano (Hermann 1976), el cual traslada cada vértice de la malla poligonal a la posición media de sus vértices vecinos, pudiéndose fijar algunos vértices para que no cambien su posición tras el suavizado. Se consideran vértices vecinos de un vértice de la malla aquellos que están unidos a éste mediante un borde de cualquiera de las caras de la malla. Este proceso admite recursividad, es decir, puede repetirse varias veces para lograr un mayor nivel de suavizado. El resultado es una malla con la misma cantidad de caras que la original con una forma más relajada. A la hora de aplicar el suavizado laplaciano a la malla que envuelve a la estructura alámbrica, se fijan los vértices de ésta que se sitúan en sus extremos superior e inferior quedando el resto libre para reubicarse. Tras el suavizado, se puede observar que los vértices de la malla que forman los nudos de la estructura tienden a expandirse, mostrando un aspecto menos anguloso que en la malla original. Puede ocurrir que la estructura alámbrica presente nudos en forma de codo en los que confluyan únicamente 2 segmentos de línea. En este caso, el suavizado laplaciano puede provocar el desplazamiento de la parte de la malla que envuelve al codo y hacer que el nudo de la estructura alámbrica quede fuera de ésta.

No obstante, el suavizado laplaciano está limitado por la resolución de la malla, es decir, la cantidad de caras que tiene la malla. Para superar esta limitación, se aplica a la malla la segunda operación de suavizado: la subdivisión de Catmull-Clark (Catmull y Clark 1978). Este proceso divide cada cara de la malla en otras 4 caras, a la vez que redefine la posición de sus vértices. El resultado es una nueva malla con un número de caras 4 veces superior a la original. La subdivisión de Catmull-Clark también admite recursividad, si bien no se aconseja aplicar el proceso muchas veces pues la resolución de la malla aumenta exponencialmente y puede prolongar en exceso el tiempo de procesado.

El objetivo de aplicar sobre la malla poligonal estos procesos de suavizado es asimilar la envolvente de la estructura alámbrica a la geometría de un árbol (Fig. 10), donde las transiciones entre el tronco y las ramas ocurren de manera suave, adaptando la curvatura de su superficie para lograr una mejor distribución de tensiones (Mattheck 1990). 

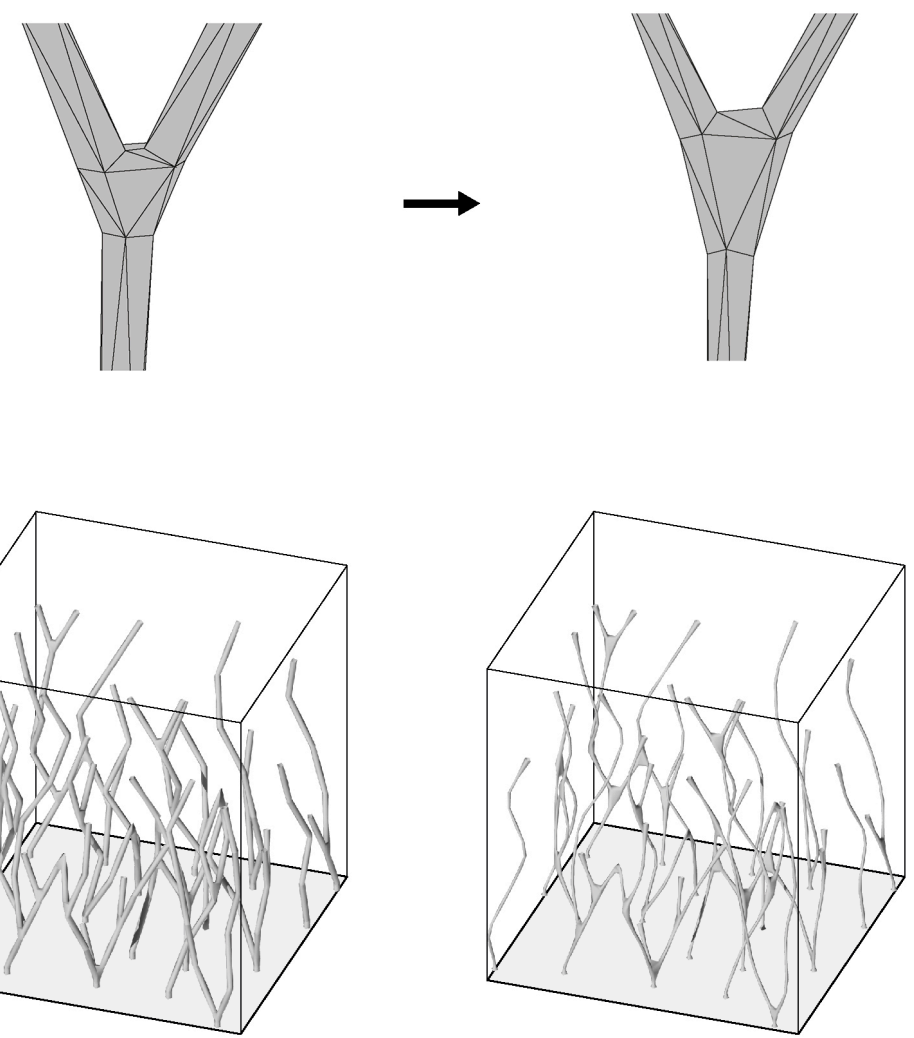

(b)
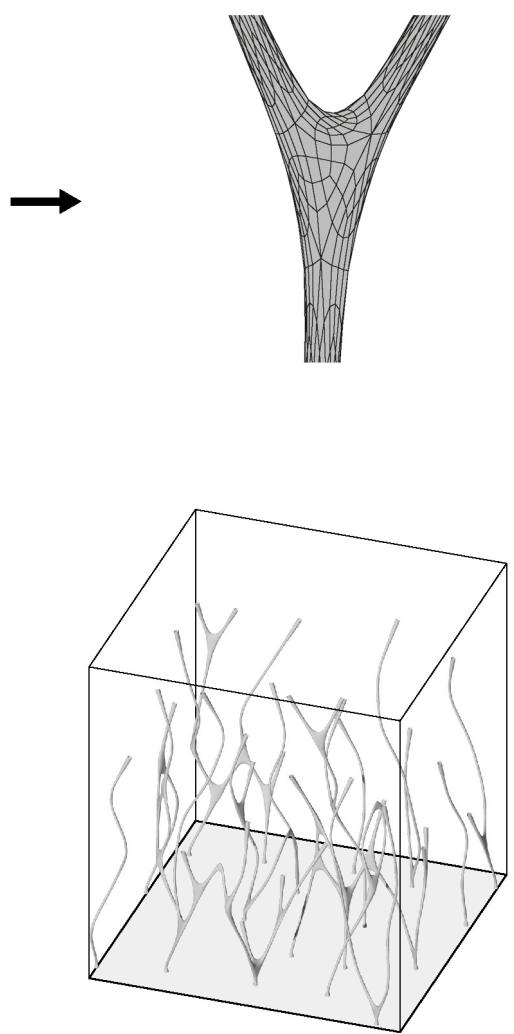

(c)

Fig. 10. Generación de la superficie que envuelve a la estructura alámbrica: aplicación de métodos Solidifyng Wireframe (a), suavizado laplaciano (b), y subdivisión de Catmull-Clark (c)

A continuación, se han realizado dos variantes de la rutina, con el objetivo de explorar el potencial de ésta para generar patrones DLA con distribuciones de material menos uniformes. En la primera de las variantes se ha puesto el valor de distancia de captura $r$ en función del número de iteraciones $t$, de manera que se cumpla la expresión

$$
r=30 / t
$$

Se puede intuir por la relación inversa entre $r$ y $t$ que la capacidad de las partículas captoras para atrapar partículas en movimiento se reduce a medida que avanza la rutina. De igual modo, la pendiente máxima de los segmentos de línea que forman el patrón DLA con respecto a la superficie base se incrementa conforme se suceden las iteraciones de la rutina. Esto se traduce en un mayor incremento de la cantidad de las partículas agregadas en las primeras iteraciones, concentrando el material en la zona del cuerpo agregado más cercana a la superficie base.

Otra de las modificaciones que se han llevado a cabo sobre este modelo, es el incremento de grosor en la malla envolvente de la estructura alámbrica, en relación con la altura a la que se sitúan los segmentos de línea que la forman. Si bien en el anterior modelo el grosor que genera la malla envolvente es constante, en este se reduce a medida que el modelo crece en una dirección perpendicular a la superficie base. Así, los segmentos de línea más alejados de la base estarán envueltos por una malla de grosor menor al que aparece en la zona más cercana a la base.

En la segunda variante, se ha procedido de igual manera que en el caso previo, pero sustituyendo la expresión anterior por la siguiente

$$
r=t+7
$$

Se deduce de la expresión que la capacidad de las partículas captoras para atrapar partículas en movimiento 
aumenta a medida que avanza la rutina, al contrario de lo que ocurre en el caso previo. De la misma forma, la cantidad de las partículas agregadas en las primeras iteraciones es menor que en las últimas, lo cual repercute en una mayor concentración de material en la parte superior del cuerpo agregado, al contrario de lo que ocurre en la variante anterior.
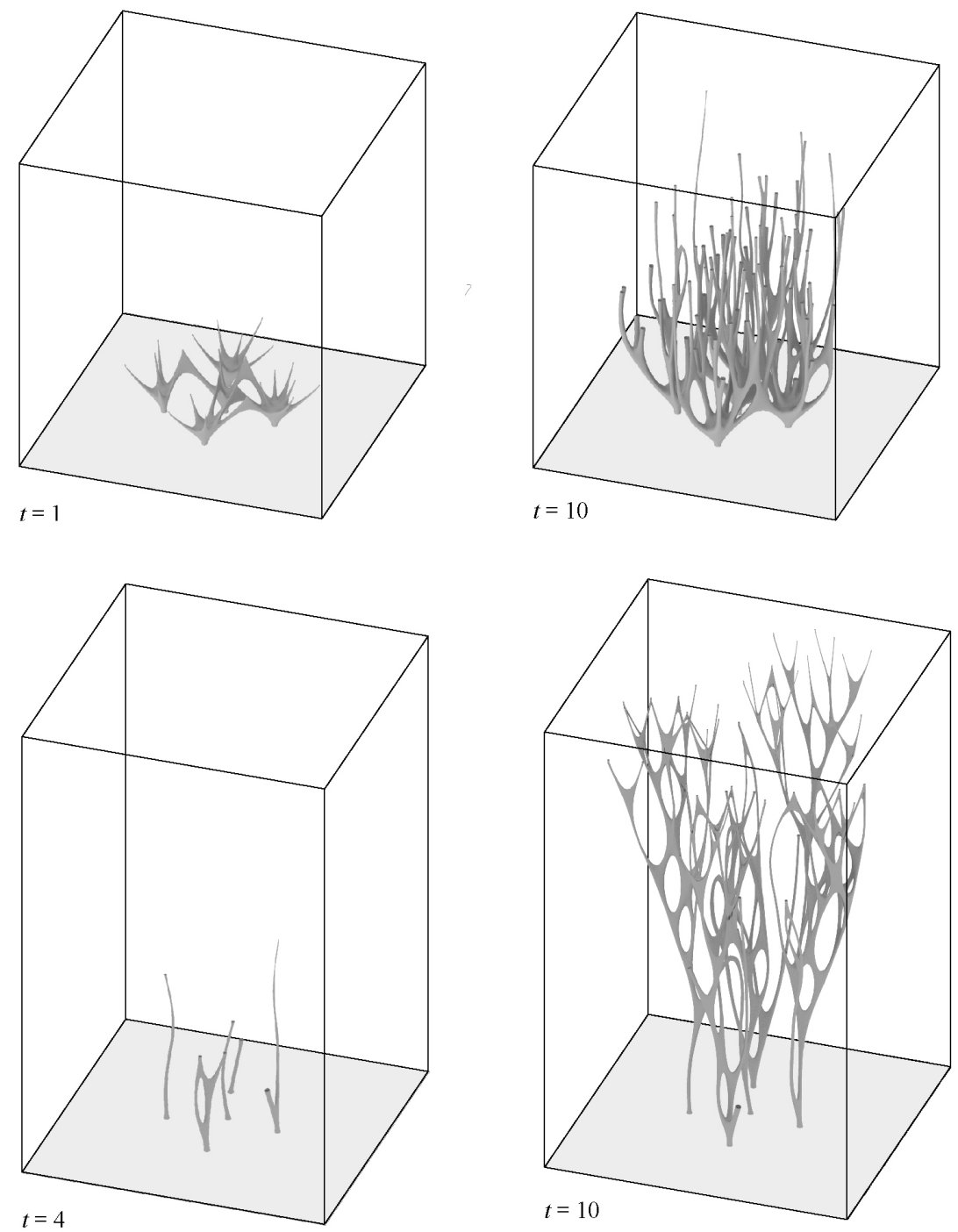

Fig. 11. Evolución del cuerpo agregado durante la ejecución de la rutina de la primera variante para $n=5, n^{\prime}=100$ y $h=30$ (arriba); ídem de la segunda variante para $n=5, n^{\prime}=100$ y $h=30$ (abajo)

\section{Discusión}

En la práctica arquitectónica convencional, los procesos de diseño se basan en la toma de decisiones por parte de un equipo humano, fiándose la conveniencia de las soluciones adoptadas a sus conocimientos e intuición. No obstante, la naturaleza ha resultado una fuente de inspiración a la hora de resolver ciertos problemas, dado que en ella existen especies que han desarrollado atributos optimizados para la supervivencia. Dado ese amplio conocimiento construido mediante siglos de selección natural, se entiende como pertinente el experimentar con procesos de diseño alternativos en los que se delegue en favor de algoritmos basados en modelos naturales la toma de decisiones sobre aspectos formales, topológicos, estructurales, etc. Además, estos sistemas complejos tienen una mayor capacidad para responder a situaciones cambiantes (Nikolov 2007).

La asimilación del cuerpo agregado a una estructura alámbrica es algo que también aparece en el trabajo de 
(Busch et al. 2011), si bien en éste las partículas agregadas adquieren la forma de elementos estructurales homogéneos que permiten ensamblarse entre sí. Estos elementos constan de unos ejes en forma de tetraedro que restringen la dirección en la que se pueden conectar, y están envueltos también por una superficie que hace que las transiciones entre elementos sean suaves. Esta condición de modularidad no es compartida por el modelo propuesto, pues en éste se permite la agregación de partículas por debajo de una distancia de captura y no a una distancia fija, de manera que los ángulos relativos entre los elementos agregados y las longitudes de éstos no son uniformes.

En otro proyecto similar, (Mayoral 2015) plantea un método de generación de estructuras mediante la agregación de unos componentes modulares cuya geometría permite formar estructuras habitables, generar superficies de fachada o albergar jardines verticales. Del mismo modo, el modelo propuesto permite cambiar las dimensiones del dominio tridimensional en el que se opera, e incluso variar a dirección de las partículas en movimiento, mostrando así una buena adaptabilidad a diferentes situaciones y contextos.

Uno de los aspectos incorporados al modelo, el de las transiciones suaves entre las ramas de la estructura, es un recurso que ha sido utilizado en otros proyectos como el vMESH Pavilion de la SUTD de Singapur, en el que se propone un método para resolver la singularidad de cada nudo estructural respondiendo a los ángulos relativos entre elementos lineales (Raspall y Bañón 2016). Esto tiene relación con el concepto de redondez de Mario Carpo, quien explica que el desarrollo de las nuevas tecnologías de diseño y fabricación han tenido un gran impacto sobre las formas arquitectónicas, de manera que ya no se aplican las rígidas leyes de la producción mecánica en un mundo donde la variación es la norma, como ocurre en la naturaleza (Carpo 2009).

En la investigación realizada, se han propuesto modificaciones sobre el modelo DLA para generar cuerpos agregados que crecen en perpendicular al suelo, añadiendo así condicionantes a las reglas de crecimiento y agregación. Esta estrategia es efectiva a la hora de orientar el crecimiento de la estructura hacia una situación concreta, y es empleada en los estudios de (Bourke 2004), en los cuales se aplican unas restricciones o condicionantes al crecimiento para que el cuerpo agregado se desarrolle sobre superficies, adoptando su forma. Además, al igual que ocurre en el presente trabajo, la estructura DLA es representada como elementos sólidos que se asemejan a raíces o ramas.

\section{Conclusiones}

El modelo propuesto ha sido construido con herramientas de diseño paramétrico que permiten su manipulación ágil y dinámica. Los parámetros editables permiten la adecuación de las estructuras ramificadas a multiplicidad de situaciones espaciales distintas. Esto hace que el modelo sea bastante flexible pese a que opere sobre un espacio tridimensional prismático. No obstante, las condiciones de contorno podrían ser fácilmente modificables hacia formas menos regulares.

Se han implementado reglas de agregación variables en el tiempo y se han mostrado las diferencias que éstas pueden producir en los resultados. Esto supone una novedad con respecto a los proyectos con los que se ha comparado previamente.

Se ha dotado al modelo DLA de tridimensionalidad y direccionalidad, dos condiciones necesarias para abordar cualquier problema de diseño estructural. También se ha establecido una distancia constante entre capas de partículas agregadas, lo cual se corresponde con la mayoría de casos prácticos en la arquitectura, en los que los niveles entre plantas están separados por la misma distancia. Estas condiciones hacen que los productos que genera el modelo puedan ser considerados para su implementación en casos reales.

Se ha asimilado el cuerpo agregado producido por el modelo a las superficies curvas y transiciones entre ramas que se dan en las plantas, mediante la implementación de una sucesión de métodos de modelado, suavizado y subdivisión de mallas poligonales. Esto no sólo tiene una justificación estructural, como se ha explicado en el artículo, sino que además supone, en combinación con el modelo DLA modificado, un procedimiento alternativo para el modelizado de estructuras arborescentes. Es en esta asociación entre estructura y significado donde el modelo se equipara a las estrategias cibernéticas, en las que la construcción es parte de un plan (Pask 2009).

Como posible trabajo futuro, se plantea incorporar criterios de resistencia mecánica en el modelo propuesto con el objetivo de condicionar el crecimiento de las estructuras ramificadas a las solicitaciones inducidas por cargas externas. Otra posible vía de continuación es el estudio de los nudos surgidos en el proceso de generación de la superficie envolvente como elementos estructurales independientes que puedan ser ensamblados con otro tipo de elementos lineales. En cualquier caso, se entiende el presente trabajo como un experimento inicial extensible 
en múltiples direcciones dentro de la investigación de sistemas complejos aplicados al diseño de estructuras.

\section{Bibliografía.}

NERDINGER, Winfried "Frei Otto. Complete Works" Birkhauser, 2005.

JENCKS, Charles. The Architecture of the Jumping Universe: A Polemic: How Complexity Science is Changing Architecture and Culture. London: Academy Editions, 1997. ISBN: 0-471-97748-9

HOLLAND, John H. Adaptation in Natural and Artificial Systems: An Introductory Analysis with Applications to Biology, Control and Artificial Intelligence. Cambridge: MIT Press, 1992. ISBN:0262082136

FRAZER, John. "Un modelo natural para la arquitectura. La naturaleza del modelo evolutivo". En: ORTEGA, L. (ed.) La digitalización toma el mando. Barcelona: Gustavo Gili, 2009. p. 29-38.

FROMM, Jochen. The Emergence of Complexity. Kassel: Kassel Univ. Press, 2004. ISBN: 3-89958-069-9

GODIN, Christophe. "Representing and encoding plant architecture: A review". Annals of Forest Science. 2000, vol 57, núm. 5, p. 413-438.

PRUSINKIEWICZ, Przemyslaw; LINDEMAYER, Aristid. The algorithmic beauty of plants. New York: Springer, 1990. 228 p. ISBN 978-1-4613-8476-2

GODIN, Christophe; COSTES, Evelyne; SINOQUET, Hervé. "A Method for Describing Plant Architecture which Integrates Topology and Geometry". Annals of Botany. 1999, vol 84, núm. 3, p. 343-357.

YAN, Hong-Ping; KANG, Meng Zhen; DE REFFYE, Philippe; DINGKUHN, Michael. "A Dynamic, Architectural Plant Model Simulating Resource-dependent Growth". Annals of Botany. 2004, vol 93, núm. 5, p. 591-602.

QUAN, Long; TAN, Ping; ZENG, Gang; YUAN, Lu; WANG, Jingdong; KANG, Sing Bing. "Image-based plant modeling“. ACM Trans.on Graphics (SIGGRAPH). 2006. vol 25, núm. 3, p. 772-778.

NEUBERT, Boris; FRANKEN, Thomas; DEUSSEN, Oliver. "Approximate image-based tree-modeling using particle flows". ACM SIGGRAPH 2007 papers on - SIGGRAPH 07. 2007, vol 26, núm. 3.

OKABE, Makoto; OWADA, Shigeru; IGARASHI, Takeo. "Interactive Design of Botanical Trees using Freehand Sketches and Example-based Editing". Computer Graphics Forum. 2005, vol 24, núm. 3, p. 487-496.

ANASTACIO, Fabricio; SOUSA, Mario C.; SAMAVATI, Faramarz; JORGE, Joaquim A. "Modeling plant structures using concept sketches". En: Proceedings of the 3rd international symposium on Non-photorealistic animation and rendering - NPAR 06.

RIAN, lasef M.; SASSONE, Mario. "Tree-inspired dendriforms and fractal-like branching structures in architecture: A brief historical overview". Frontiers of Architectural Research. 2014, vol 3, núm. 3, p. 298-323.

NERDINGER, Winfried. Frei Otto, complete works: lightweight construction natural design. Basel: Birkhäuser, 2005. ISBN: 3764372311

VON BUELOW, Peter. "A Geometric Comparison of Branching Structures in Tension and Compression versus Minimal Paths". En: Proceeding of IASS 2007. (Venecia 3-6 de diciembre de 2007).

GAWELL, Ewelina. "Non-Euclidean Geometry in the Modelling of Contemporary Architectural Forms". The Journal of Polish Society for Geometry and Engineering Graphics. 2013, vol 24, p. 35-43.

WITTEN, Thomas A.; SANDER, Leonard M. "Diffusion-limited aggregation". Physical Review B. 1983, vol 27, núm 9, p. 5686-5697.

TURCOTTE, Donald L.; PELLETIER, Jon D.; NEWMAN, William I. "Networks with Side Branching in Biology". Journal of Theoretical Biology. 1998, vol 193, núm. 4, p. 577-592.

NetLogo, 1999, Center for Connected Learning and Computer-Based Modeling, Northwestern University, Evanston, IL. : U. Wilensky.

NetLogo DLA model, 1997, Center for Connected Learning and Computer-Based Modeling, Northwestern Uni- 
versity, Evanston, IL. : U. Wilensky.

NetLogo DLA Alternate model, 2005, Center for Connected Learning and Computer-Based Modeling, Northwestern University, Evanston, IL. : U. Wilensky.

NetLogo DLA Alternate Linear model, 2005, Center for Connected Learning and Computer-Based Modeling, Northwestern University, Evanston, IL. : U. Wilensky.

Rhinoceros 3D, 2012, Robert McNeel \& Associates.

Grasshopper 3D, 2014, Robert McNeel \& Associates.

Exoskeleton, 2014, D. Piker y D.Stasiuk.

SRINIVASAN, Vinod; MANDAL, Esan; AKLEMAN, Ergun. "Solidifying Wireframes".En: Proceedings of the 2004 bridges conference on mathematical connections in art, music, and science.

Weaverbird, 2012, G. Piacentino.

HERMANN, Leonard R. "Laplacian-isoparametric grid generation scheme". Journal of the Engineering Mechanics Division. 1976, vol 105, núm. 5, p. 749-756.

Laplacian smoothing, 2016. En.wikipedia.org [online]. Recuperado de https://en.wikipedia.org/wiki/Laplacian smoothing

CATMULL, Edwin; CLARK, Jim. "Recursively generated B-spline surfaces on arbitrary topological meshes". Computer-Aided Design. 1978, vol 10, núm 6, p. 350-355.

Catmull-Clark subdivision surface, 2016. En.wikipedia.org [online]. Recuperado de https://en.wikipedia.org/wiki/Catmull-Clark_subdivision_surface

MATTHECK, Claus. "Engineering components grow like trees". Mat.-wiss. u. Werkstofftech. 1990, vol 21, núm 4, p. 143-168.

NICOLOV POPOV, Nicolay. "How can NetLogo be used in the landscape architectural design process?" Master of Landscape Architecture, Unitec New Zealand, 2007.

BOURKE, Paul. "Constrained Diffusion Limited Aggregation in 3 Dimensions". Computer and Graphics. 2006, vol 30, núm. 4, p. 646-649.

BUSCH, Benjamin; LADURNER, Georg; BAHARLOU, Ehsan; MENGES, Achim. "Adaptive Structure: A Modular System for Generative Architecture”. En: GA2011 - XIV Generative Art Conference.

MAYORAL, Eduardo. Arquitecturas biosintéticas: la acción arquitectónica a través de la ingeniería de lo vivo y lo no-vivo. Lucena: Recolectores Urbanos, 2015. ISBN: 8494168428

RASPALL, Felix; BAÑÓN, Carlos. "vMESH : How to print Architecture?". En: SIGraDi 2016, XX Congreso de la Sociedad Ibero-americana de Gráfica Digital. (Buenos Aires 9-11 de noviembre de 2016).

CARPO, Mario. " La desaparición de los idénticos. La estandarización arquitectónica en la era de la reproductibilidad digital". En: ORTEGA, L. (ed.) La digitalización toma el mando. Barcelona: Gustavo Gili, 2009. p. 59-66.

PASK, Gordon. " La significación arquitectónica de la cibernética". En: ORTEGA, L. (ed.) La digitalización toma el mando. Barcelona: Gustavo Gili, 2009. p. 15-28.

RIAN, lasef Md; SASSONE, Mario. "Tree-inspired dendriforms and fractal-like branching structures in architecture: A brief historical overview” Frontiers of Architectural Research, 2014. Vol 3, issue 3, pp 298-323.

OTTO, Frei, RASCH, Bodo "Finding form", Axel Menges 1995. P157-166.

GORDON J.E. "The Science of Structures and Materials”. Scientific American Library, 1988. Pp 161-174.

FALK, A., BUELOW, P.V. "Combined timber plate and branching column systems-variations and development of system interaction". In: Proceedings of the International Association for Shell and Spatial Structures (IASS) Symposium. 2009. Valencia.

BUELOW, P. "A Geometric Comparison of Branching Structures in Tension and in Compression versus Minimal Paths". 2007. 


\section{Biografías}

Salvador Serrano Salazar. Arquitecto por la Escuela Politécnica Superior de la Universidad de Alicante (2013). Becario de colaboración MECD en el Departamento de Expresión Gráfica y Cartografía (2012-2013) y profesor honorífico de Proyectos Arquitectónicos (2014), en la Universidad de Alicante. Ha participado en proyectos que han sido reconocidos con el Premio Nacional de Educación (2013), otorgado por el MECD; la Mejor Práctica en el X Concurso Internacional de Buenas Prácticas Ciudadanas (2014) otorgado por la ONU; y el Segundo Premio Nacional de Arquitectura Efímera (2016), otorgado por Veredictas Excellence Standards. Actualmente, desarrolla una investigación centrada en el estudio de sistemas complejos y su aplicación al diseño estructural, apoyándose en herramientas open source y técnicas de autoproducción como la fabricación digital.

José Carrasco Hortal. Departamento de Expresión Gráfica, Composición y Proyectos. Profesor Titular. Miembro de grupo de investigación "Proyectos Arquitectónicos: Pedagogías Críticas, Políticas Ecológicas y Prácticas Materiales (PAPCPEPM)", UA. Producción investigadora en: representación compleja de la arquitectura; codiseño y facilitación para la arquitectura doméstica; aprendizaje por acción; diseño basado en modelos naturales; cartografías inmersivas en el paisaje; arquitectura transmodal. Cofundador en 2011 de "Common Extra House Lab" y en 2013 del grupo "Viceversos Socioarquitectónicos".

Francesc Morales Menárguez, Estudiante de doctorado en Ingeniería de Materiales, Estructuras y Terreno: Construcción Sostenible. Arquitecto por la Escuela Politécnica Superior de la Universitat d'Alacant (2015) e Ingeniero Técnico Agrícola por la Universitat Miguel Hernández d'Elx (1998). Colaborador honorífico de la asignatura "Estructura del Proyecto" de la titulación de Arquitectura de la Universitat d'Alacant en el año 2016-2017. Sus investigaciones incluyen la participación en la red sobre la docencia "Viceversos" y la atención a nuevas tecnologías para conseguir arquitecturas interactivas. Es coautor de diferentes artículos y presentación en congresos en el estudio de mecanismos de sistemas emergentes. Coordinador y profesor del taller internacional "Worldmaking and Technoledge" entre las universidades de Alicante, TuDelft (Países Bajos) y York (Canadá). 